\title{
Correlation between osteosarcoma and the expression of WWOX and p53
}

\author{
PINGTAO LIU ${ }^{1 *}$, MINGYUE WANG ${ }^{2 *}$, LI LI $^{1}$ and TAO JIN ${ }^{3}$ \\ ${ }^{1}$ Department I of Orthopedics, Jingmen No. 2 People's Hospital; ${ }^{2}$ Jingmen Red Cross Blood Center; \\ ${ }^{3}$ Department III of Orthopedics, Jingmen No. 2 People's Hospital, Jingmen, Hubei 448000, P.R. China
}

Received April 4, 2017; Accepted August 2, 2017

DOI: $10.3892 / \mathrm{ol} .2017 .6747$

\begin{abstract}
The objective of this study was to analyze the effect of the expression of WWOX and p53 on the growth of MG-63 osteosarcoma cells and to explore the correlation between osteosarcoma and the expression of WWOX and p53. WWOX and p53-overexpressing MG-63 osteosarcoma cell lines were established by transfection and named the MW and MP cell lines, respectively. Untransfected MG-63 cells (blank control) were used as control. Quantitative polymerase chain reaction (qPCR) and western blot analysis were used to detect the expression of WWOX and wild-type p53 mRNA and protein, respectively. The effects of WWOX and p53 (wild-type) on the activity of MG-63 cells were determined by MTT assay and flow cytometry. The expression of mutant p53 protein in 65 cases of osteosarcoma was detected by immunohistochemistry to analyze the correlation between p53 and the development of osteosarcoma. qPCR showed that WWOX and p53 mRNA was overexpressed in MW and MP cells, respectively. Western blot analysis showed that the levels of WWOX and p53 protein in MW and MP cells were higher than in the blank control group. MTT assay showed that the cell proliferation ability of MW and MP cells was significantly lower than in the blank control group. Flow cytometry showed that $78.49 \%$ of MW and $66.76 \%$ of MP cells were arrested in the G0/G1 phase. Immunohistochemistry showed that mutant p53 was highly expressed in osteosarcoma, with a positive expression rate of $47.7 \%$. The expression rate was positively correlated with the pathological grade of cancer. In conclusion, WWOX can affect the cell cycle of MG-63 osteo-
\end{abstract}

Correspondence to: Dr Li Li, Department I of Orthopedics, Jingmen No. 2 People's Hospital, 39 Xiangshan Road, Jingmen, Hubei 448000, P.R. China

E-mail:ilj79j@163.com

Dr Tao Jin, Department III of Orthopedics, Jingmen No. 2 People's Hospital, 39 Xiangshan Road, Jingmen, Hubei 448000, P.R. China

E-mail: j2a474@163.com

${ }^{*}$ Contributed equally

Key words: WWOX gene, p53 gene, osteosarcoma sarcoma cells to inhibit cell proliferation, which provides new insights into gene therapy for osteosarcoma. The two types of the p53 gene have different functions in the development of osteosarcoma. Wild-type p53 acts as a tumor suppressor, while mutant p53, which is overexpressed in malignant osteosarcoma, has a carcinogenic effect associated with the degree of osteosarcoma.

\section{Introduction}

Osteosarcoma, one of the primary malignant tumors of bone, is the most common bone cancer and accounts for $20 \%$ of primary malignant tumors (1). Osteosarcoma occurs mainly in youth, with a higher incidence in males (2). At present, the treatment of osteosarcoma primarily includes surgery, radiotherapy and chemotherapy and the 5-year survival rate of osteosarcoma ranges from 20 to $75 \%$ (3). However, there are many chemotherapy-insensitive osteosarcoma patients with a high risk of tumor metastasis and recurrence. Therefore, it is highly important to identify a more effective and stable method for the diagnosis and treatment of osteosarcoma. The activation of proto-oncogenes and the inactivation of tumor suppressor genes can affect the cell cycle and apoptosis, which in turn lead to tumorigenesis (4). Therefore, studying osteosarcoma-related tumor suppressor genes at the molecular level to improve the diagnosis and treatment of osteosarcoma has high clinical value.

Tumor suppressor genes can inhibit tumor cell proliferation, oncogenesis, invasion, metastasis, apoptosis and even death and have become a research focus in the field of osteosarcoma gene therapy. The WWOX and p53 genes are classical tumor suppressor genes. WWOX, which is located on chromosome 16q23, spans the entire chromosomal fragile site, FRA16D (5). WWOX contains nine exons. The first four exons of WWOX encode the WW domain and exons 5-8 encode the oxidoreductase domain (6). WWOX can inhibit tumor cell activity and this inhibitory effect has been reported in a variety of tumors, such as glioma (7), lung cancer (8) and thyroid cancer (9). The p53 gene, which is located on the short arm of human chromosome 17, encodes a protein that can promote DNA repair, transient and sustained cell growth arrest, apoptosis and senescence (10). There are two types of the p53 gene, wild-type and mutant. Wild-type p53, which has anticancer effects, can cause apoptosis to eliminate tumor cells (11). The 
common mutation site of mutant p53 is position 143 (from A to $\mathrm{G}$ ) and mutant p53 can cause tumorigenesis (12).

The function of the WWOX gene in osteosarcoma has not been well studied and comparison studies of the wild-type and mutant p53 genes are also rare. In the present study, we examined the effects of stable expression of the WWOX and p53 genes in transfected cell lines. The effects of the WWOX and p53 (wild-type) genes on the proliferation and cell cycle of osteosarcoma cells were assessed by MTT assay and flow cytometry to investigate the relationship between osteosarcoma and the expression of the WWOX and p53 (wild-type) genes. Additionally, the expression of the mutant p53 gene in osteosarcoma tissue was detected by immunohistochemistry to investigate the relationship between the mutant p53 gene and the degree of malignancy of osteosarcoma.

\section{Materials and methods}

Reagents. The human osteosarcoma cell line, MG-63, was purchased from the Institute of Basic Medical Sciences of the Chinese Academy of Medical Sciences (Beijing, China); plasmids were constructed at the early stage of this project by the team members; Lipofectamine ${ }^{\circledR} 2000$ was from Invitrogen; Thermo Fisher Scientific, Inc. (Waltham, MA, USA); MTT and RIPA lysis buffer both from Beijing PPLYGEN Technology Co., Ltd. (cat. no. C1053; Beijing, China); TRIzol kit was from Invitrogen; Thermo Fisher Scientific, Inc. (cat. no. 15596026); TransScript ${ }^{\circledR}$ One-Step gDNA Removal and cDNA Synthesis SuperMix kit both from TransGen Biotech Co., Ltd. (cat. no. AT311-02; Beijing, China); FastStart Universal SYBR-Green Master (Rox) kit was from Roche Diagnostics (cat. no 04913914001; Indianapolis, IN, USA); BCA kit was from Thermo Labsystems (cat. no 23225; Santa Rosa, CA, USA); Cell cycle assay kit for flow cytometry was from Baomanbio Co., Ltd. (cat. no. GMS10021.1; Shanghai, China).

Mouse monoclonal wild-type p53 antibody (dilution, 1:500; cat. no. 178924), mouse monoclonal mutant p53 antibody (dilution, 1:500; cat. no. 178379) and HRP-labeled goat anti-mouse secondary antibody (dilution, 1:2,000; cat. no. 197302) were from Beijing Zhongshan Golden Bridge Biotechnology Co., Ltd. (Beijing, China).

Construction of WWOX and p53-overexpressing MG-63 cell lines through transfection. The recombinant eukaryotic overexpression plasmids of WWOX and p53 were, respectively transfected into MG-63 osteosarcoma cells by liposome transfection to establish cell lines that stably expressed WWOX and p53. These cell lines were named the MW and MP cell line, respectively.

Cell culture. MG-63 cells were cultured in MEM medium supplemented with $10 \%$ fetal bovine serum (FBS). G418 was added to DMEM medium containing $10 \%$ FBS to a final concentration of $1,000 \mu \mathrm{g} / \mathrm{ml}$ for culture of transfected MW and MP cells. The cells were cultured at $37^{\circ} \mathrm{C}$ with $5 \% \mathrm{CO}_{2}$. When cells were $80-90 \%$ confluent, they were subcultured according to the required dilution ratio.

Quantitative polymerase chain reaction ( $q P C R) . \mathrm{MW}, \mathrm{MP}$ and MG-63 cells under healthy growth conditions were collected at the logarithm growth period. Total cellular RNA was extracted using TRIzol reagent and RNA was reverse transcribed into cDNA according to the instructions of the TransScript ${ }^{\circledR}$ One-Step gDNA Removal and cDNA Synthesis SuperMix kit. The primer sequences of WWOX, p53 (wild-type) and the endogenousv GAPDH gene were designed with reference to a large number of studies (Table I). All primers were synthesized by a third party. qPCR was carried out using the FastStart Universal SYBR-Green PCR Master (Rox) kit and the relative expression of each gene was calculated using the $2^{-\Delta \Delta \mathrm{Cq}}$ method.

Western blot analysis. MW, MP and MG-63 cells under healthy growth conditions were collected at the logarithm growth period. Cells were lysed with lysis buffer and total protein was extracted. Total protein concentration was determined using a BCA kit. Protein samples were subjected to (SDS-PAGE), after which protein was transferred to PVDF membranes using a semi-dry method. The membranes were blocked with 5\% skimmed milk, followed by incubation with primary mouse monoclonal wild-type p53 antibody (dilution, 1:500; cat. no. 178924) overnight at $4^{\circ} \mathrm{C}$, after washing. Membranes were then incubated with HRP-labeled goat anti-mouse secondary antibody (dilution, 1:2,000; cat. no. 197302) for $1 \mathrm{~h}$ at room temperature. After washing, a gel imaging system was used to detect protein signals. $\beta$-actin was used as the endogenous control and Image J software was used to quantify protein expression.

MTT assay. MW, MP and MG-63 cells under healthy growth conditions were collected at the logarithm growth period and digested with trypsin to prepare cell suspensions. Cells in suspension were seeded in 96-well plates and MTT reagent was added 1-6 days later. After adding the MTT reagent, cells were cultured at $37^{\circ} \mathrm{C}$ with $5 \% \mathrm{CO}_{2}$ for an additional $4 \mathrm{~h}$, followed by the addition of DMSO to stop the reaction. The 96-well plates were placed in a microplate reader and the absorbance value of each well was measured at $490 \mathrm{~nm}$ to calculate the survival rate and construct the growth curve.

Flow cytometry. MW, MP and MG-63 cells were digested with trypsin, followed by centrifugation to remove the medium and obtain cell pellets. The cell cycle assay kit for flow cytometry and the flow cytometer were used for the cell cycle analysis.

Immunohistochemistry. The expression of mutant p53 in osteosarcoma was detected by immunohistochemistry. A total of 65 paraffin-embedded osteosarcoma tissue samples were selected in Jingmen No. 2 People's hospital. The samples were diagnosed by pathological diagnosis and were classified into different differentiation levels: High, 17 cases; moderate, 25 cases; and low differentiation, 23 cases. Paraffin-embedded osteosarcoma tissue samples were sectioned at a thickness of $5 \mu \mathrm{m}$. Immunohistochemical staining was performed using the SP method (13) to detect the expression of mutant p53 protein in osteosarcoma tissues with different pathological grades. PBS was used as the negative control of the primary antibody. A double-blind method was used to observe the signals. Five high-power microscopic fields were randomly selected for observation. Brown particles within the nucleus represented 
Table I. Primer sequences.

\begin{tabular}{lcc}
\hline Genes & Forward $\left(5^{\prime}-3^{\prime}\right)$ & Reverse $\left(5^{\prime}-3^{\prime}\right)$ \\
\hline WWOX & TCCTCAGAGTCCCATCGATTT & CGGCAGCAGTTGTTGAAGTA \\
p53 & TACTCCCCTGCCCTCAACAAG & CGCTATCTGAGCAGCGCTCAT \\
GAPDH & CATGCCATCACTGCCACCCA & TTGACAAAGTGGTCGTTGAG
\end{tabular}

Table II. Criteria for the determination of mutant p53 expression.

The proportion of positive cells $(\%)$

Classification

$$
<5
$$

$5-25$

$26-50$

$+$

$>50$

positive signals. Specific criteria for the determination of mutant p53 expression are shown in Table II.

Statistical analysis. Data are presented as percentage and one-way ANOVA was performed using SPSS 13.0 statistical software (SPSS, Inc., Chicago, IL, USA) for comparisons between groups. $\mathrm{P}<0.05$ was considered to indicate a statistically significant difference.

\section{Results}

Expression of WWOX and p53 (wild-type) mRNA in the three groups of cells. qPCR analysis showed that the expression of WWOX mRNA was increased in MW cells compared with the blank control group and the expression of p53 (wild-type) mRNA was increased in MP cells compared with the blank control group (Fig. 1).

Expression of WWOX and p53 (wild-type) protein in the three groups of cells. Western blot analysis showed that the expression of WWOX and p53 (wild-type) protein in the transfected cells was higher than those in the control group and the increased protein expression was consistent with the increased mRNA expression (Fig. 2).

The effect of WWOX and p53 (wild-type) on the proliferation of MG-63 cells as determined by MTT assay. The cell survival rates of MW and MP cells at 1-6 days after the beginning of incubation are shown in Table III and the growth curve is shown in Fig. 3. The survival rates of MW and MP cells were significantly lower than those of the blank control group at each time point $(\mathrm{P}<0.05)$. As shown in Table III and Fig. 3, the two genes showed significant inhibitory effects on MG-63 osteosarcoma cells at 3 days after the beginning of incubation.

Changes of the cell cycle after WWOX and p53 (wild-type) gene transfection. Flow cytometric analysis showed that MW
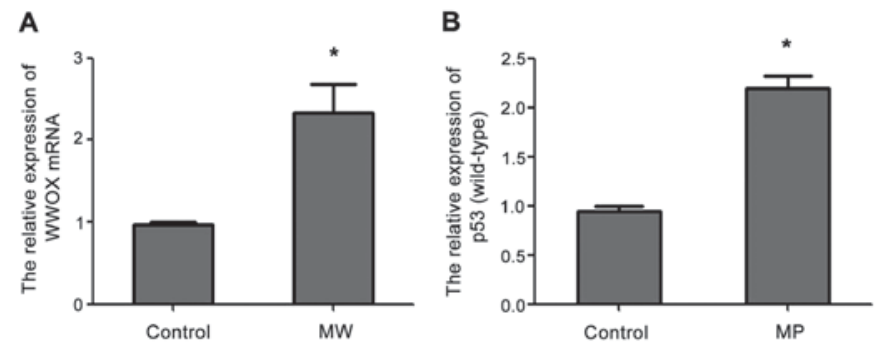

Figure 1. The expression of WWOX and wild-type p53 mRNA. qPCR analysis showed that the expression of (A) WWOX mRNA was increased in MW cells compared with the blank control group and the expression of (B) wild-type p53 mRNA was increased in MP cells compared with the blank control group. ${ }^{*}$ Compared with the control group, $\mathrm{P}<0.05$.
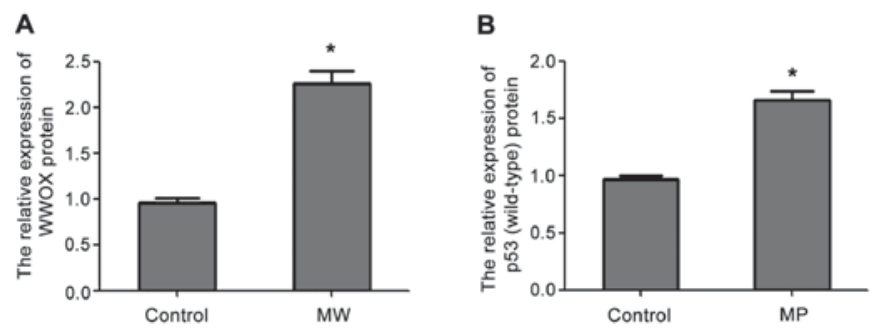

C
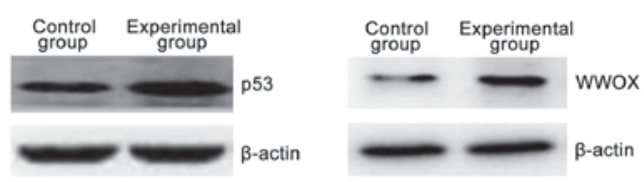

Figure 2. (A and B) Expression of WWOX and wild-type p53 protein in MW and MP cells, respectively. (C) Western blot analysis showed that the expression of WWOX protein in the MW cell line was significantly higher than in the control group and the expression of wild-type p53 protein in the MP cell line was significantly higher than in the control group. Representative western blot analysis. Comparison of the relative protein levels between the control group and experimental groups. Compared with the control group, ${ }^{*} \mathrm{P}<0.05$.

and MP cells were blocked in the G0/G1 phase, suggesting that overexpression of the WWOX and p53 (wild-type) genes causes cell cycle arrest of MG-63 osteosarcoma cells at the G0/G1 phase (Table IV).

Immunohistochemical analysis of the expression of mutant 553 in osteosarcoma tissue. The mutant p53 protein is located in the nucleus and the brown particles observed after immunohistochemical staining represented positive signals. Microscopic observation showed that the positive expression rate of mutant p53 protein in 65 cases of osteosarcoma was $47.7 \%$ and the 
Table III. Survival rates of MW and MP cells (\%).

\begin{tabular}{lcccccc}
\hline Days of incubation & 1 & 2 & 3 & 4 & 5 & 6 \\
\hline Blank control group & 100 & 100 & 100 & 100 & 100 & 100 \\
MW & $92^{\mathrm{a}}$ & $82^{\mathrm{a}}$ & $54^{\mathrm{a}}$ & $63^{\mathrm{a}}$ & $74^{\mathrm{a}}$ & $81^{\mathrm{a}}$ \\
MP & $90^{\mathrm{a}}$ & $84^{\mathrm{a}}$ & $59^{\mathrm{a}}$ & $69^{\mathrm{a}}$ & $72^{\mathrm{a}}$ & $74^{\mathrm{a}}$ \\
\hline
\end{tabular}

${ }^{a}$ Compared with the blank control at each time point, $\mathrm{P}<0.05$.

Table IV. The cell cycle of MW and MP cells (mean \pm standard deviation).

\begin{tabular}{lccr}
\hline Groups & $\mathrm{G}_{0} / \mathrm{G}_{1}(\%)$ & $\mathrm{S}(\%)$ & \multicolumn{1}{c}{$\mathrm{G}_{2} / \mathrm{M}(\%)$} \\
\hline Control & $24.07 \pm 1.235$ & $45.49 \pm 0.987$ & $25.45 \pm 0.912$ \\
MW & $78.49 \pm 2.392$ & $12.01 \pm 1.209$ & $8.30 \pm 0.462$ \\
MP & $66.76 \pm 0.235$ & $19.96 \pm 0.689$ & $11.34 \pm 0.764$ \\
\hline
\end{tabular}

Table V. The expression status of p53 (mutant) protein in each pathological grade of osteosarcoma.

\begin{tabular}{lcccr}
\hline Pathological grade & Case & $\begin{array}{c}\text { Positive } \\
\text { (cases) }\end{array}$ & $\begin{array}{c}\text { Negative } \\
\text { (cases) }\end{array}$ & $\begin{array}{r}\text { Positive } \\
\text { rate }(\%)\end{array}$ \\
\hline High differention & 17 & 5 & 12 & 29.4 \\
Moderate differention & 25 & 10 & 15 & 40.0 \\
Low differention & 23 & 16 & 7 & 69.6 \\
$\begin{array}{l}\text { Total positive } \\
\text { expression rate }\end{array}$ & & & & 47.7 \\
\hline
\end{tabular}

positive expression rate was related to pathological grade. According to the criteria for determination, the expression status of the mutant $\mathrm{p} 53$ protein in each pathological grade of osteosarcoma is summarized in Table $\mathrm{V}$ and the positive expression rates of the mutant $\mathrm{p} 53$ protein in each pathological grade are shown in Fig. 4. The highest positive expression rate of mutant p53 was found in low differentiation osteosarcoma tissue and the positive expression rate of mutant $\mathrm{p} 53$ protein decreased with increased degree of tumor malignancy.

\section{Discussion}

The diagnosis and treatment of osteosarcoma at the genetic level is an area of intense research. Studies have shown that tumor suppressor genes can inhibit tumorigenesis and tumor growth (14). Therefore, in the present study, we investigated the functions of two classic tumor suppressor genes, p53 and WWOX, at the molecular level to explore the correlation between the two genes and osteosarcoma.

WWOX is a tumor suppressor gene with a length of approximately 1.1 Mb. Studies have shown that the WWOX gene plays an important role in preventing tumorigenesis and tumor growth. WWOX can function as a transcription factor to regulate tumor metastasis and growth by regulating the
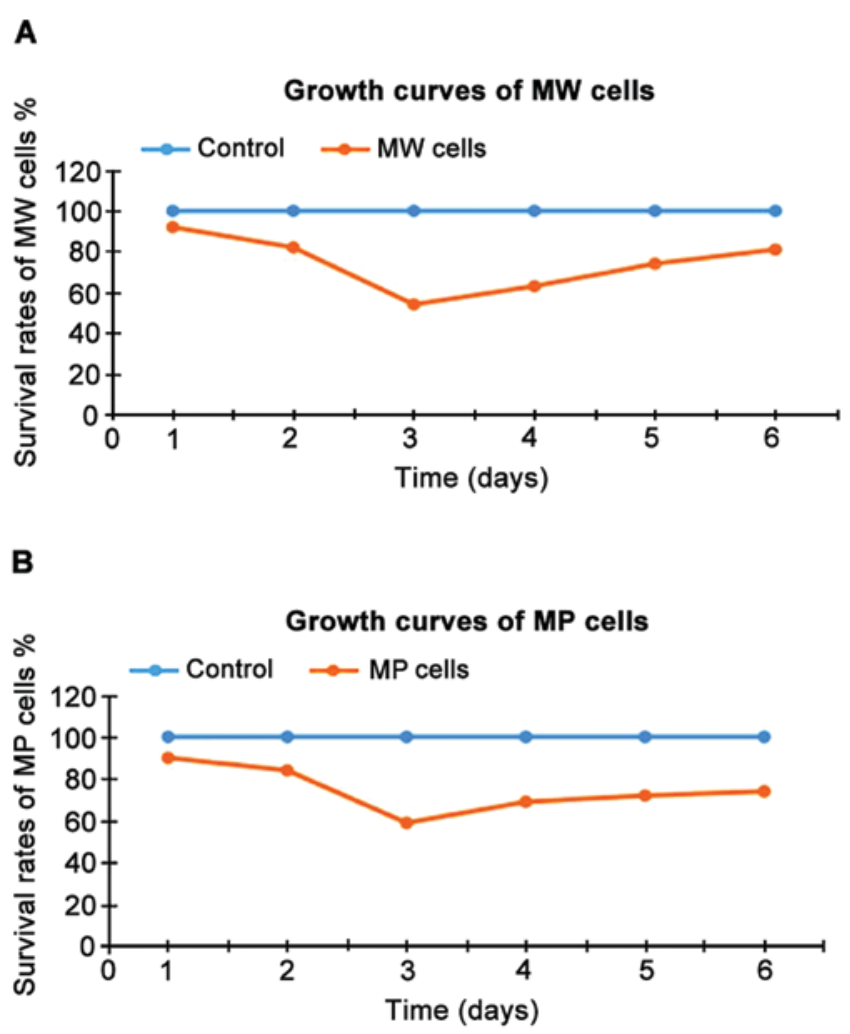

Figure 3. Growth curves of MW and MP cells. The results of MTT assay showed that the WWOX gene inhibited the proliferation of MG-63 osteosarcoma cells; (A) the wild-type $\mathrm{p} 53$ gene inhibited the proliferation of MG-63 osteosarcoma cells; (B) after 3 days of incubation, significant inhibitory effects of these genes on MG-63 osteosarcoma cells was observed. Compared with the blank control at each time point, $\mathrm{P}<0.05$.

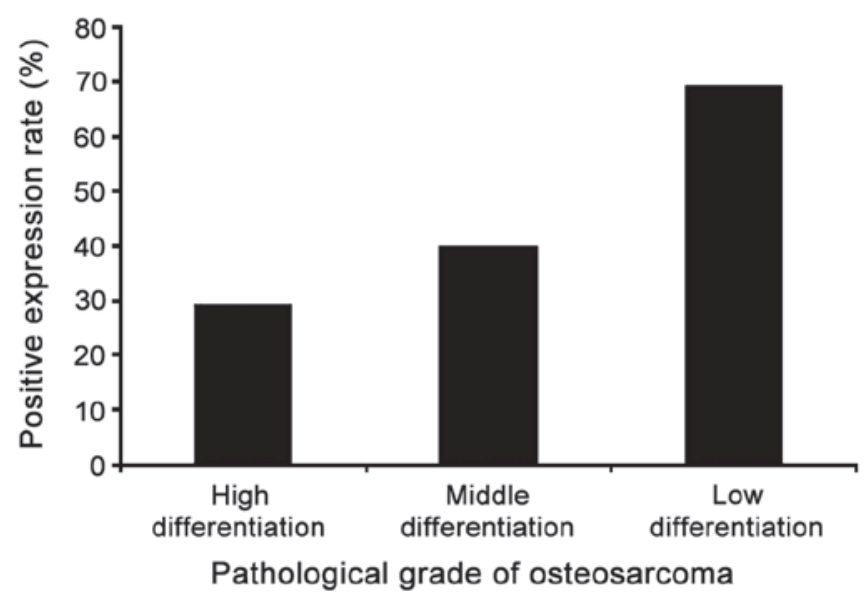

Figure 4. The positive expression rate of p53 according to osteosarcoma pathological grade. 
expression of various genes (15) and reduced expression of the WWOX gene can cause tumorigenesis and promote the growth of osteosarcoma (16).

P53 is another well-known tumor suppressor gene. The main role of p53 is to induce apoptosis and affect the cell cycle (17). The p53 gene exists as two types - wild and mutant and the two types have different functions (18). Because of the short half-life, the p53 (wild-type) protein cannot be easily detected by immunohistochemistry $(19,20)$. However, mutant p53 can be detected by immunohistochemistry because of a long half-life. In this study, p53 (wild-type) and mutant p53 were studied. The two transfected MG-63 osteosarcoma cell lines, which stably expressed the WWOX and p53 (wild-type) genes, were constructed and named the MW and MP cell lines, respectively. Measurement of the levels of mRNA and protein in the two groups showed that WWOX and p53 were stably expressed in the cell lines and the two groups of transfected cells were therefore suitable for use in subsequent experiments. Based on the successful establishment of the MW and MP cell lines, we simultaneously studied the effects of the WWOX and p53 (wild-type) genes on MG-63 osteosarcoma cells via in vitro experiments. The growth rates of MG-63 cells transfected with the WWOX or p53 (wild-type) gene overexpression plasmids were reduced and flow cytometric analysis showed that overexpression of the WWOX and p53 (wild-type) genes can arrest MG-63 cells in the G0/G1 phase of the cell cycle. These results indicate that the WWOX and p53 (wild-type) genes can inhibit the proliferation of osteosarcoma cells by affecting the cell cycle.

Immunohistochemical analysis showed that the wild-type and mutant p53 genes have completely opposite functions. Mutant p53 was highly expressed in osteosarcoma and its expression was positively related to the pathological grade of osteosarcoma.

In conclusion, detection of the expression of the WWOX and p53 genes has great clinical value in the diagnosis, prevention and treatment of osteosarcoma. The WWOX gene can effectively prevent the growth of osteosarcoma, which is beneficial for the diagnosis and treatment of the disease. The p53 gene exists as two different types, where the wild-type p53 gene has the same function as WWOX in osteosarcoma, and the mutant p53 gene has important clinical value in the determination of the degree of malignancy of osteosarcoma.

\section{References}

1. Chen X, Bahrami A, Pappo A, Easton J, Dalton J, Hedlund E, Ellison D, Shurtleff S, Wu G, Wei L, et al: Recurrent somatic structural variations contribute to tumorigenesis in pediatric osteosarcoma. Cell Rep 7: 104-112, 2014.

2. Mirabello L, Troisi RJ and Savage SA: Osteosarcoma incidence and survival rates from 1973 to 2004. Cancer 115: 1531-1543, 2009.
3. Anninga JK, Gelderblom H, Fiocco M, Kroep JR, Taminiau AH, Hogendoorn PC and Egeler RM: Chemotherapeutic adjuvant treatment for osteosarcoma: where do we stand? Eur J Cancer 47: 2431-2145, 2011.

4. Clark JC, Dass CR and Choong PF: A review of clinical and molecular prognostic factors in osteosarcoma. J Cancer Res Clin Oncol 134: 281-297, 2008

5. Abu-Odeh M, Salah Z, Herbel C, Hofmann TG and Aqeilan RI: Wwox, the common fragile site FRA16D gene product, regulates ATM activation and the DNA damage response. Proc Natl Acad Sci USA 111: E4716-E4725, 2014.

6. Chang NS, Pratt N, Heath J, Schultz L, Sleve D, Carey GB and Zevotek N: Hyaluronidase induction of a WW domain-containing oxidoreductasethat enhances tumor necrosis factor cytotoxicity. J Biol Chem 276: 3361-70, 2001.

7. Yu K, Fan J, Ding X, Li C, Wang J, Xiang Y and Wang QS Association study of a functional copy number variation in the Wwox gene with risk of gliomas among Chinese people. Int J Cancer 135: 1687-1691, 2014.

8. Huang D, Qiu F, Yang L, Li Y, Cheng M, Wang H, Ma G, Wang Y, $\mathrm{Hu} \mathrm{M}, \mathrm{Ji} \mathrm{W}$, et al: The polymorphisms and haplotypes of Wwox gene are associated with the risk of lung cancer in southern and eastern Chinese populations. Mol Carcinog 52: E19-E27, 2013.

9. Cancemi L, Romei C, Bertocchi S, Tarrini G, Spitaleri I, Cipollini M,Landi D, Garritano S, Pellegrini G, Cristaudo A, et al: Evidences that the polymorphism Pro-282-Ala within the tumor suppressor gene Wwox is a new risk factor for differentiated thyroid carcinoma. Int J Cancer 129: 2816-2824, 2011.

10. Wu Z, Ma C, Shan Z, Ju Y, Li S and Zhao Q: Histone deacetylase inhibitors suppress the growth of human osteosarcomas in vitro and in vivo. J Buon 18: 1032-1037, 2013.

11. Levine AJ and Oren M: The first 30 years of p53: Growing ever more complex. Nat Rev Cancer 9: 749-757, 2009.

12. Li D and Marchenko ND: ErbB2 inhibition by lapatinib promotes degradation of mutant p53 protein in cancer cells. Oncotarget 8: 5823-5833, 2017.

13. Chen W, Cooper TK, Zahnow CA, Overholtzer M, Zhao Z, Ladanyi M, Karp JE, Gokgoz N, Wunder JS, Andrulis IL, et al: Epigenetic and geneticloss of HIC1 function accentuates the role of p53 in tumorgenes. Cancer Cell 6: 387-398, 2004.

14. Chang NS, Hsu LJ, Lin YS, Lai FJ and Sheu HM: WW domain-containingoxidoreductase: A candidate tumor suppressor. Trends Mol Med 13: 12-22, 2007.

15. Del MS and Aqeilan RI: Tumor suppressor Wwox inhibits osteosarcoma metastasis by modulating RUNX2 function. Sci Rep 5: 12959, 2015.

16. Abdeen SK, Del MS, Hussain S, Abu-Remaileh M, Salah Z, Hagan J, Rawahneh M, Pu XA, Russell S, Stein JL, et al: Conditional inactivation of the mouse Wwox tumor suppressor gene recapitulates the null phenotype. J Cell Physiol 228: 1377-1382, 2013.

17. Mercer J, Mahmoudi M and Bennett M: DNA damage, p53, apoptosis and vascular disease. Mutat Res 621: 75-86, 2007.

18. Subramanian M, Jones MF and Lal A: Long non-codingRNAs embedded in the $\mathrm{Rb}$ and p53 pathways. Cancers (Basel) 5: 1655-1675, 2013.

19. Bargonetti J and Manfred IJJ: Multiple roles of the tumor suppressor p53. Curr Opin Oncol 14: 86-91, 2002.

20. Mardanpour K, Rahbar M and Mardanpour S: Coexistence of HER2, Ki67 and p53 in Osteosarcoma: A strong prognostic factor. N Am J Med Sci 8: 210-214, 2016. 